\title{
Sea Surface Conditions Remotely Sensed by Upward-Looking ADCPs
}

\author{
MARTIN ViSBECK* AND JÜRGEN FISCHER \\ Institut für Meereskunde, Kiel, Germany
}

(Manuscript received 8 January 1994, in final form 28 June 1994)

\begin{abstract}
Surface data obtained from $153-\mathrm{kHz}$ acoustic Doppler current profilers deployed in the Greenland Sea at about 350-m depth during the winter of $1988 / 89$ were investigated under several aspects. First a method is described to improve the instrument depth measurements using the binned backscattered energy profile near the surface. The accuracy of the depth estimates is found to be significantly better than $0.5 \mathrm{~m}$.

Further, improvements of wind speed estimates were found by using the ambient noise in the $150-\mathrm{kHz}$ band in favor of the surface backscattered energy as suggested by Schott. Limitations of the ambient sound method at low wind speeds are presented when thermal noise overwhelms the wind-induced noise.

Finally, a method to detect the presence of sea ice above the ADCP is presented by cross correlating the surface backscatter strength and the magnitudes of all Doppler velocity components. The resulting time series of ice concentration are in overall good agreement with Special Sensor Microwave/Imager estimates but allow for higher temporal resolution. Further, in the vicinity of the ice edge, enhanced high-frequency ambient noise in the $150-\mathrm{kHz}$ band was observed.
\end{abstract}

\section{Introduction}

Acoustic Doppler current profilers (ADCPs) have been used successfully over the past decade to measure ocean currents remotely up to a distance of $300 \mathrm{~m}$ from the instruments in either shipboard (Joyce et al. 1982 ) or moored applications (Schott 1986). The objectives of most of the moored applications for ADCPs are to measure velocity profiles with high accuracy. Therefore, as many pings as possible are averaged to one ensemble, which then is stored internally. More recently it was recognized that the near surface data from upward-looking instruments provide additional information on wind speed and direction (Schott 1989; Brown et al. 1992). Both studies showed the dependence of the surface backscattered energy on wind speed and the Doppler direction resemblance of the wind direction.

Upward-looking ADCPs have been used successfully to measure ice drift using the bottom-track mode (Belliveau et al. 1990). Since this method requires additional energy for bottom-track pings, it is not well suited for long-term deployments with emphasis on accurate velocity profiling. Here we describe a method that al-

\footnotetext{
* Current affiliation: Center of Meteorology and Physical Oceanography, Department of Atmospheric, Earth and Planetary Science, MIT, Cambridge, MA 02139.
}

Corresponding author address: Dr. Martin Visbeck, Institut für Meereskunde, Düsternbrooker Weg 20, Kiel D-24105, Germany. lows ice detection and ice velocity measurements using the standard profiles without extra bottom-track pings.

Because ADCPs are more or less upward-looking sonars, they can be used to determine the distance to the surface. The sea surface is a strong acoustical target and easy to detect in the backscattered energy profiles. The drawback is the range-gated signal of the ADCPs, which restricts the resolution to the bin length to typically between 4 and $16 \mathrm{~m}$. A method (section $3 \mathrm{a}$ ) is presented that overcomes this restriction and increases the resolution of the distance measurements significantly.

We will revisit remote wind measurements as proposed by Schott (1989) and improve the results by replacing the strength of the surface backscatter in favor of ambient noise records (section 3b). Further, a multiparameter ice-detection scheme is presented and compared to Special Sensor Microwave/Imager (SSM/I) ice concentrations (section 3c).

\section{Instrumentation and dataset}

From June 1988 to June 1989 four moorings with ADCPs were deployed in the Greenland Sea (Schott et al. 1993). Two of these were upward-looking (moorings 319 and 250) and were used for this investigation. The ADCPs in moorings $\mathrm{T} 5$ and $\mathrm{T} 6$ were either downward-looking or deployed too deep (at $1400 \mathrm{~m}$ in T6) to measure up to the surface. Mooring 319 was deployed in the central Greenland Sea at $75^{\circ} \mathrm{N}$, $5^{\circ} \mathrm{W}$, while the position of mooring 250 was $73^{\circ} 22^{\prime} \mathrm{N}$, $0^{\circ} 48^{\prime} \mathrm{W}$ at the southeastern periphery of the Greenland Sea gyre (Fig. 1a). Both moorings had 200-m-long 


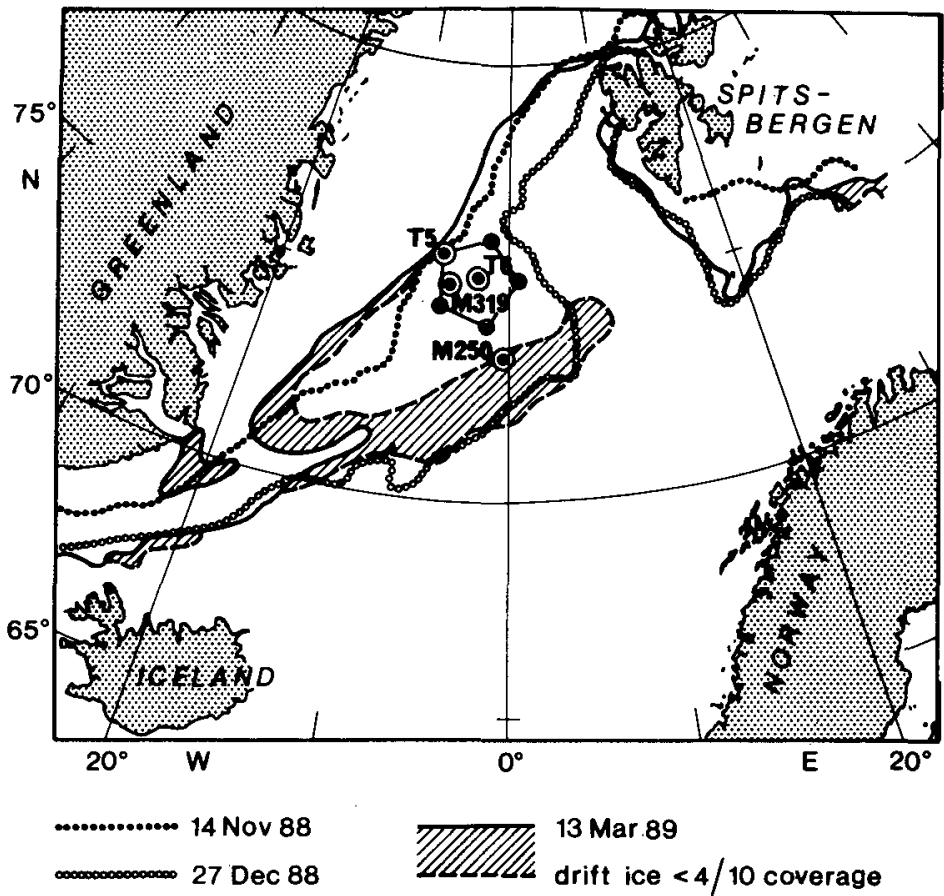

(a)

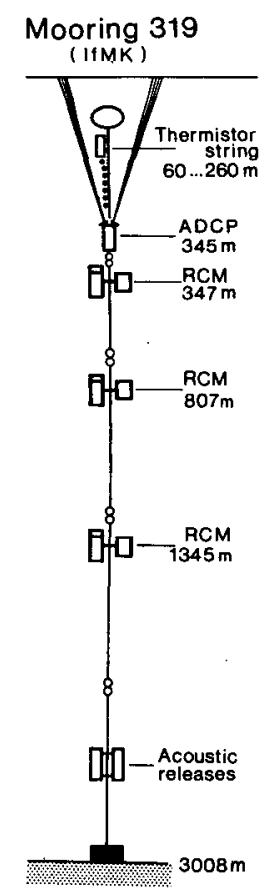

(b)

FIG. 1. Position of moored ADCPs during winter 1988/89 in the Greenland Sea. (a) The marginal ice zone for three different phases is included. (b) The design of mooring 319 (mooring 250 was similar).

thermistor strings in the top $300 \mathrm{~m}$, followed by an upward-looking ADCP at about 350-m depth with an Aanderaa current meter (ACM) directly underneath the ADCP for intercomparison and two additional ACM's in deeper layers (Fig. 1b).

The ADCPs were RD-Instruments $153-\mathrm{kHz}$ profilers with $20^{\circ}$ beam angle and were programmed to measure velocity profiles in three components: east-west $(U)$, north-south $(V)$, and vertical $(W)$. While the vertical velocity is an average over two independent measurements from the two orthogonal beam pairs, the "error" velocity $(\mathrm{Ve})$ is the difference of the two. The latter should be zero in a homogeneous velocity field providing no measurement errors are involved (RDIPrimer 1989). In addition the strength of the backscattered signal for each of the four beams, the "echo amplitude" (EA) was stored. The data were ensemble averaged over 125 individual profiles, which were burst sampled every second at 30 -min intervals. The vertical resolution was $8.6 \mathrm{~m}$ ( $8-\mathrm{m}$ bins) after adjustment to sound speed and geometry $\left(20^{\circ}\right.$ beam angle). The number of bins recorded was 65 to assure that even during large mooring excursions, the surface was still within the range of the ADCP. For this set of parameters, the nominal accuracy of the horizontal velocity was $\pm 1.7 \mathrm{~cm} \mathrm{~s}^{-1}$ (RDI-Primer 1989), $\pm 0.6 \mathrm{~cm} \mathrm{~s}^{-1}$ for the vertical velocity.

Time series of raw data are depicted by Fig. 2 during the period when the marginal ice zone (MIZ) passed by station 250 in late November 1988 . The wind data are 6-h forecasts from the European Centre for Medium-Range Weather Forecasts (ECMWF) model for a grid point close to the mooring (Fig. 2a). The surface Doppler velocities are usually large during ice-free conditions (Schott 1989) and were truncated at 50 $\mathrm{cm} \mathrm{s}^{-1}$ if the speed was larger (Fig. 2b). SSM/I ice concentrations were kindly provided by the Electromagnetics Institute, Denmark (L. Toudal 1992, personal communication; Fig. 2c). The ADCP surface data, such as target strength (that is, EA corrected for transmission loss; RDI-Primer 1989), horizontal speed, vertical velocity, and error velocity, are displayed below the ADCP ice indicator (Figs. 2d-h). Ambient noise in the $150-\mathrm{kHz}$ band (Fig. $2 \mathrm{i}$ ) is measured $0.3 \mathrm{~s}$ later than the arrival from the surface and potentially contains information on wind speed (Vagle et al. 1990) and ice noise (Waddell and Farmer 1988).

\section{Methods \\ a. Instrument depth}

The depth of upward-looking ADCPs can be derived directly from the acoustical measurements by evaluating the local maximum of the backscattered signal similar to upward-looking sonars or inverted echo sounders. A typical profile of backscattered energy as a function of time or slant range relative to the sea surface is shown in Fig. 3. The main peak of enhanced 

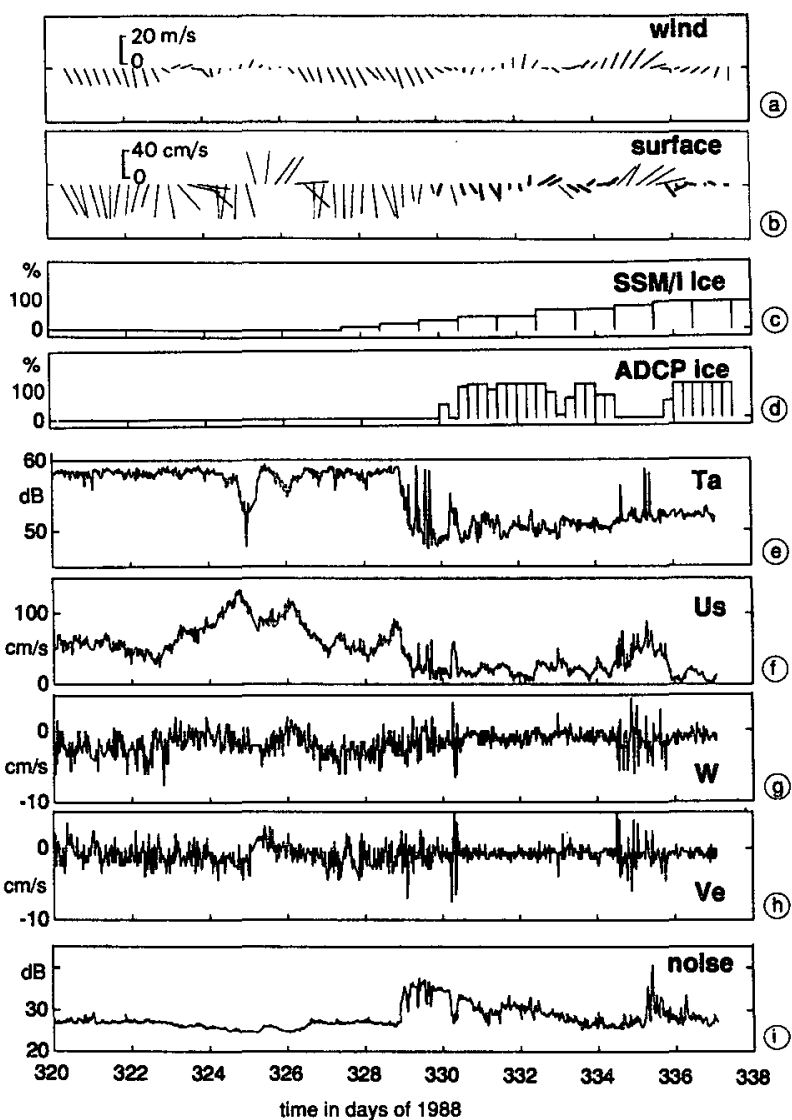

FlG. 2. Time series from station 250 when the ice edge passed over the mooring location from 15 October to 3 December 1989 . (a) ECMWF bottom-layer wind speed. (b) ADCP surface bin Doppler velocities. (c) SSM/I daily ice concentration. (d) ADCP ice indicator (6-h average). ADCP surface bin data (e) mean target strength, (f) horizontal velocity speed, $(\mathrm{g})$ vertical velocity speed, and $(\mathrm{h})$ error velocity. (i) ADCP recorded ambient noise $0.3 \mathrm{~s}$ after the surface return (bin 65).

target strength is associated with the sea surface. A secondary maximum occurs due to vertically traveling sidelobe reflections, which appear before the main lobe, since the distance straight upward is shorter than the slanted path of the main beam. Below the sidelobe bin, the volume backscatter is recorded. After the mainlobe backscatter has arrived, weaker reflections from the side lobes were received, but ultimately after some time the ADCP recorded only the ambient noise.

In the binned data, as stored by the ADCPs, the profile of the surface backscatter is only crudely sampled. A first estimate of the distance from the transducer heads to the surface is given by the bin number with maximum target strength. This yields a time series of distances with a resolution of one bin length, for example, 4-16 m depending on parameter settings. The surface-reflected energy is distributed over more than one bin, however, and the broadness of the surface peak (Fig. 3) will be used to increase the resolution of the depth estimate. The width of the surface peak is larger than the pulse length (here equal to bin length) due to slanted beams $\left(20^{\circ}\right.$ to vertical ) and beam aperture of approximately $4^{\circ}$. To make sure that more than one cell of the binned profile is affected by the surface peak, the bin length should be smaller than the half-width $h_{s}$ of the surface peak. The latter can be estimated by geometric considerations as a function of beamwidth $\beta$, tilt $\alpha$, and instrument depth $H$ :

$$
h_{s}=H \frac{\sin \alpha \sin \beta}{\cos (\alpha+\beta) \cos (\alpha-\beta)} \text {. }
$$

For an ADCP with $20^{\circ}$ beam angle and $4^{\circ}$ beam aperture, this yields $h_{s} / H=0.027$. Multiplied by the instrument depth of $350 \mathrm{~m}$, the minimal width of the surface energy peak $\left(h_{s}= \pm 9.5 \mathrm{~m}\right)$ is just resolved by the $8.6-\mathrm{m}$ bin length. The actual width of the surface peak appeared somewhat broader (Fig. 3), either due to larger beam aperture or acoustical surface conditions such as waves and vertical-penetrating bubble clouds (Vagle and Farmer 1992).

We calculated an improved instrument depth by fitting a parabola to the near surface backscatter profiles using the following procedures.

1) Calculate target strength from the echo amplitudes to correct for beam spreading and clear-water attenuation (RDI-Primer 1989).

2) Find the maximum target strength bin for each beam.

3) Fit a parabola to three adjacent target strength values around the surface maximum.

4) Determine the maximum target strength and its slant range to the ADCP transducer heads analytically from the parabola fit.

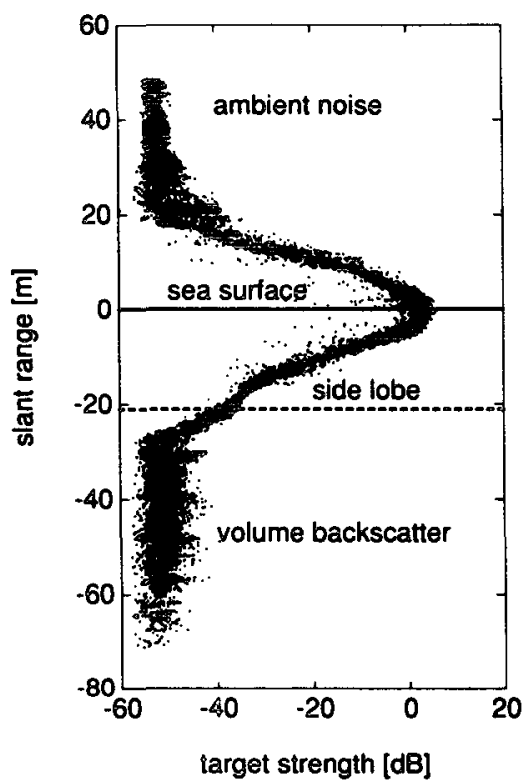

Fig. 3. Profile of near-surface backscatter strength versus slant range to the surface from 8 September to 10 October 1989 for station 319. 
5) Project the slant range to vertical distance using pitch, roll, and the harmonic mean sound speed.

The resulting four estimates of the instrument depths, one for each beam, showed some offset in the order of $0.5 \%(<2 \mathrm{~m})$ of the depth but were well correlated. The differences could be reduced by introducing a bias to the tilt sensors of the order of $1^{\circ}$. This bias is a plausible error, since the tilt meters are difficult to mount and adjust mechanically within the ADCP tranceducer head. The mean surface distance (averaged over the four beams) was consistent with pressure fluctuations recorded by the ACM $2 \mathrm{~m}$ below the ADCP (Fig. 4). The resolution of the mooring excursions was significantly higher for the ADCP than for the 1000psi pressure gauge of the ACM, which had approximately $0.7-\mathrm{db}$ resolution (Fig. 4b). The high-frequency depth fluctuations for subtidal periods yielded an rms of $12 \mathrm{~cm}$. Mooring motion within that frequency range should be small, since the subtidal current fluctuations were small and vertically uncorrelated. Therefore, this number may serve as an accuracy estimate of the depth measurements.

Limitations of the procedure were rare occasions of missing surface reflections. During these periods the energetic main-lobe signal disappeared, but an enhanced sidelobe signal remained. The latter might be used, however, instead of the main-lobe peak to estimate the instrument depth. Low wind speeds were usually found during such events, and it seemed likely that a calm sea surface led to total reflection of the tilted main lobe away from the ADCP. The acoustics involved during these times is not totally clear and beyond the scope of this report.

Summarizing, a robust and accurate procedure was developed to deduce the instrument depth from the binned "echo amplitude" profiles with an estimated accuracy of better than one-half a meter.

\section{b. Winds}

Schott (1989) has shown that the strength of the ADCP surface backscatter was correlated with wind speed, while the Doppler direction coincided with wind direction. Brown et al. (1992) had problems in deducing the wind direction from their surface Doppler directions, and one of the differences was much stronger surface currents in their case that were not wind driven. While Schott (1989) attributed the wind dependence of the surface backscatter to Bragg scattering, Brown et al. ( 1992) also discussed the effects of bubble clouds.

Our data resemble the findings of Schott (1989) with a reasonable correlation of wind direction from the ECMWF model and ADCP surface Doppler direction. The weaker magnetic field of the polar latitudes compared to the low-latitude calibration site, however, affected the quality of the ADCP direction measurements. We were able to correct for the bulk of the error using the ACM currents as a reference, but errors of
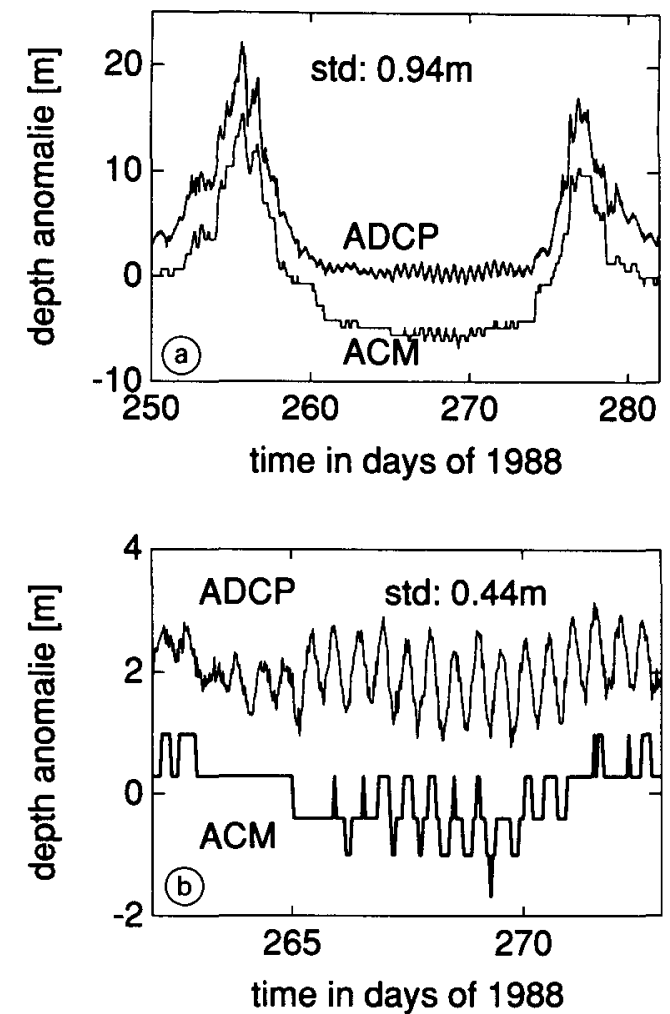

FIG. 4. Vertical mooring excursion recorded by the ACM pressure sensor and deduced from the acoustic measurements of the nearby ADCP. For better comparison the mean depth over each of the periods displayed was removed. (a) ADCP time series is offset by $5 \mathrm{~m}$. (b) Close up from 20 to 30 September 1989 with ADCP time series offset by $2 \mathrm{~m}$.

approximately $10^{\circ}$ remained (Schott et al. 1993). Therefore, we will leave a closer investigation of the wind direction versus the surface Doppler direction to more appropriate datasets.

The target strength was not as useful to represent wind speed as anticipated. A saturation level seemed to occur at higher wind speeds. We will therefore present a different signal to gain information on wind speed. Programming the ADCP such that the instrument is still listening after the excited and backscattered sound has died out offers the opportunity to measure ambient sound within the ADCP frequency band (here approximately $150 \mathrm{kHz}$ ). In theory the increased ambient noise due to wind and background thermal noise intersect somewhere between 60 and $200 \mathrm{kHz}$ (Fig. 5 , Urick 1983). Instruments designed to determine wind speed from ambient sound-for example, WCITAN (weather observations through ambient noise)-listen to the frequency band between 3 and $25 \mathrm{kHz}$ (Vagle et al. 1990) in the range of a well-defined slope of the wind noise and with a much weaker thermal noise level (Fig. 5).

Nevertheless, we inspected the time-dependent signal in bin 65 some $0.3 \mathrm{~s}$ after the surface return of the 


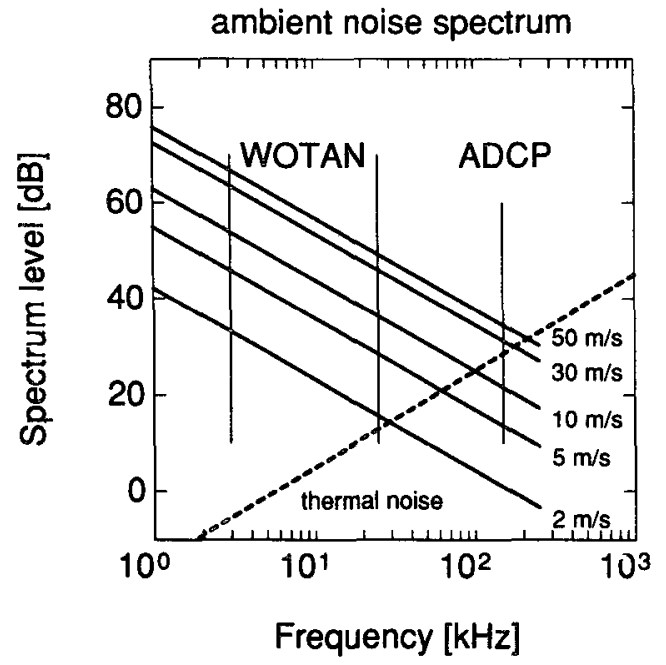

Fig. 5. Spectra of ambient noise for different wind speeds (Vagle et al. 1990) and thermal noise (Urick 1983).

stations 319 and 250 (Fig. 6). The loudest signal is attributed to ice noise (Urick 1983) and will be discussed in the next section.

During the ice-free phase, the ambient noise was well correlated (correlation 0.8 ) with the square root of ECMWF wind stress (Figs. 7a; 8a,b). A quadratic regression was applied to estimate wind stress from ambient noise yielding

$$
\tau^{1 / 2}=2.8-0.33 \text { (noise) }+9.2 \times 10^{-3}(\text { noise })^{2} .
$$

From the scatterplot it is obvious that a low-level cutoff exists in the ambient noise (Fig. 7a). This might be attributed to the constant thermal noise level and restricts the minimum wind stress detection to some 0.05 $\mathrm{Pa}$ or approximately $5 \mathrm{~m} \mathrm{~s}^{-1}$ wind speed at this instrument depth.

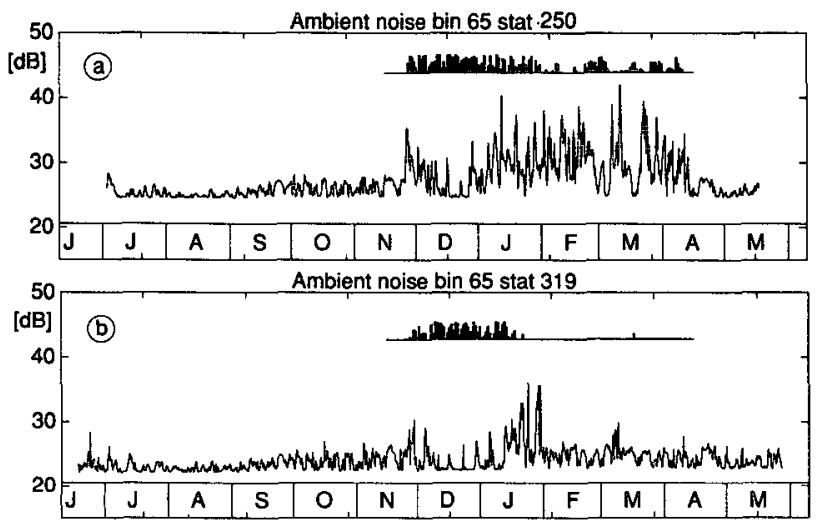

FIG. 6. Ambient noise records from bin 65 approximately $0.3 \mathrm{~s}$ after the surface reflection for (a) station 250 and (b) station 319. Daily fraction of ice is included.
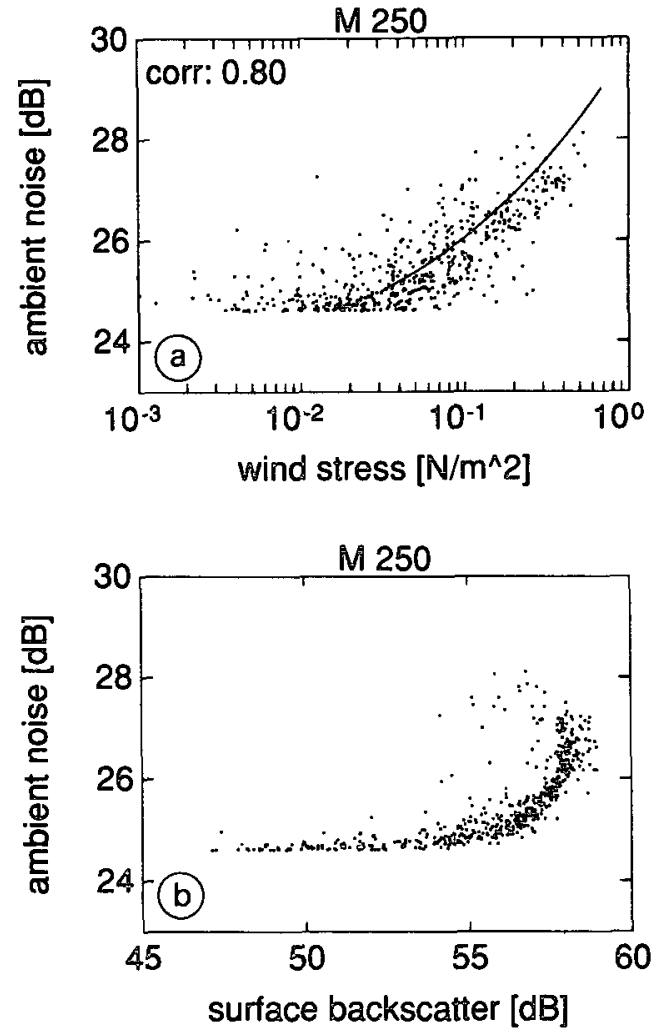

FIG. 7. Scatterplots of 6-h-averaged ECMWF wind stress data ADCP surface target strength, and ambient noise. (a) Ambient noise versus wind stress. (b) Ambient noise versus surface backscatter.

The representation of wind stress by ambient noise was significantly better than by the strength of the surface backscatter (correlation 0.5; Figs. 8a,c) using the regression suggested by Schott (1989)

$$
\log _{10}(\tau)=0.11(\text { surf })-7.48
$$

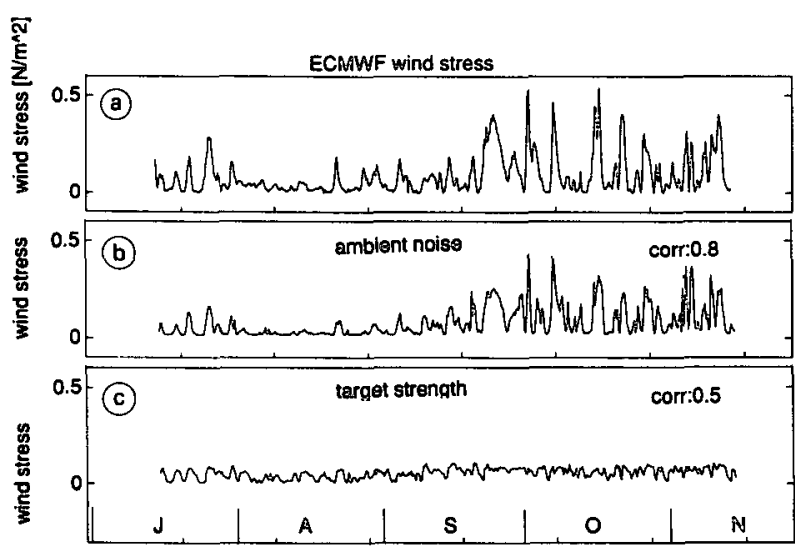

FIG. 8. Wind stress as forecasted by (a) the ECMWF model, deduced from (b) ADCP ambient noise, and (c) surface backscatter strength 
A scatterplot of ambient noise versus surface target strength enlightens two problems. At high ambient noise levels, that is, at high wind speeds, the backscatter shows a saturation level. On the other hand, low backscatter energies coincide with the ambient thermal noise cutoff.

In summary, we found an additional promising signal of wind speed in the noise level recorded by the ADCP. The acoustics in that frequency range are not well surveyed (D. Farmer 1993, personal communication ) and need closer investigation. We do not expect the accuracy of ambient noise wind speed determination with ADCPs to be as good as with the WOTAN technique, but it seems to work better than the surface backscatter for intermediate to high wind speeds.

\section{c. Ice conditions}

The general spatial extent of the ice cover can be remotely sensed by satellites using passive microwaves (Barry et al. 1993) with a typical horizontal resolution of $30 \mathrm{~km}$ and one or two scans per day. Here we present a different method to deduce the presence of ice from the ADCP sea surface signals by combining the Doppler velocity and target strength data. This "local" ice indicator might be compared to satellite ice concentrations on longer than daily timescales.

\section{1) ICE DETECTION}

The presence of ice affects several ADCP parameters (Figs. 2e-h). The most obvious signal was reduced target strength at the sea surface (Fig. 2e) at times when the SSM/I data indicated ice above the mooring. One reason for the observed energy reduction might be that some sound energy is propagating inside the ice floe and is not reflected.

Belliveau et al. (1990) showed that the surface-track, that is, the upside-down bottom-track, Doppler $W$ and error velocity $E$ were both small when there was ice above the instrument. Our data (Figs. $2 \mathrm{~g}, \mathrm{~h}$ ) support their findings, but this criterion alone did not seem to be sufficient to distinguish between ice-covered and icefree conditions during all periods. There were times during the summer when $W$ and $E$ were small, but no other evidence supported the presence of ice. Therefore, we propose a multiparameter ice indicator using the following procedures.

1) Calculate vertical and error velocity variance and highpass the signal with a 6-h cutoff.

2) Identify periods of reduced target strength due to calm wind periods.

3) Determine thresholds for each parameter to discriminate ice-free from ice-covered periods.

4) Average the ice-no-ice time series to obtain fractional ice cover, that is, daily ice concentration or percentage of ice-covered periods per day:
The threshold values were chosen by comparing two 15-day-long periods, from 15 to 30 September 1988 representing open water and from 15 to 30 December 1988 with maximum ice coverage.

The target strength TA shows a reduction of $5 \mathrm{~dB}$ when the station was ice covered (Fig. 2), and the distributions of ice-free and ice-covered periods yield a clear threshold of $55 \mathrm{~dB}$, with $99 \%$ of the ice-covered data below and $97 \%$ of the open-water data above the threshold (Fig. 9).

The speed of the horizontal surface Doppler also decreased during the ice-covered phase. The physical interpretation of the surface Doppler for open water conditions is not obvious and probably a mixture of different wave speeds in the process of Bragg scattering (Schott 1989) with typical values on the order of 100 $\mathrm{cm} \mathrm{s}^{-1}$. For the ice-covered case, Belliveau et al. (1990) had shown that the surface Doppler represents ice drift. Using speed as an ice-no-ice criteria might not be appropriate in some regions with large ice drift. For the central Greenland Sea, however, we did not expect ice drift speeds above $50 \mathrm{~cm} \mathrm{~s}^{-1}$ and therefore used 50 $\mathrm{cm} \mathrm{s}^{-1}$ as a threshold (Fig. 9b).

The variance of the vertical velocity time series was calculated with a 6-h high-pass cutoff. A threshold of $1 \mathrm{~cm}^{2} \mathrm{~s}^{-2}$ discriminated $89 \%$ of the ice data and $94 \%$ during the ice-free period (Fig. 9c). The error velocity $E$ showed roughly the same behavior as the vertical velocity, and the same threshold was used (Fig. 9d).

For comparison, the daily fraction of ADCP ice, deduced from the four different parameters, are shown together with SSM/I ice concentrations during the winter $1988 / 89$ at both stations (Fig. 10). The general ice conditions are similar in all records with differences in the details. Target strength and surface speeds show ice more often than vertical and error velocity variance. Whether this is a significant signal due to different ice properties and ice types remains an open question since no in situ ice observations were available.

It is in the hands of the user to decide which of the parameters is sufficient for his/her purpose. In our case we were interested in a conservative estimate and therefore cross correlated all four ice indicators (Fig. 10).

In general we found good agreement between ADCP and SSM/I ice concentration, keeping in mind the 20$50-\mathrm{km}$ correlation scale of the SSM/I compared to the $300-\mathrm{m}$ footprint of the ADCP beams.

\section{2) ICE DRIFT}

Since Nansen's work, it has been known that ice drifts at a rate of approximately one-fiftieth of the wind speed and about $30^{\circ}$ to the right of the wind. Linear Ekman theory yields $45^{\circ}$ deflection, while factors such as stratification and turbulence generally cause the observed turning angle to be smaller (Hunkins 1975).

Belliveau et al. (1990) have shown that ADCPs can measure ice drift using bottom-track mode. No bottom 

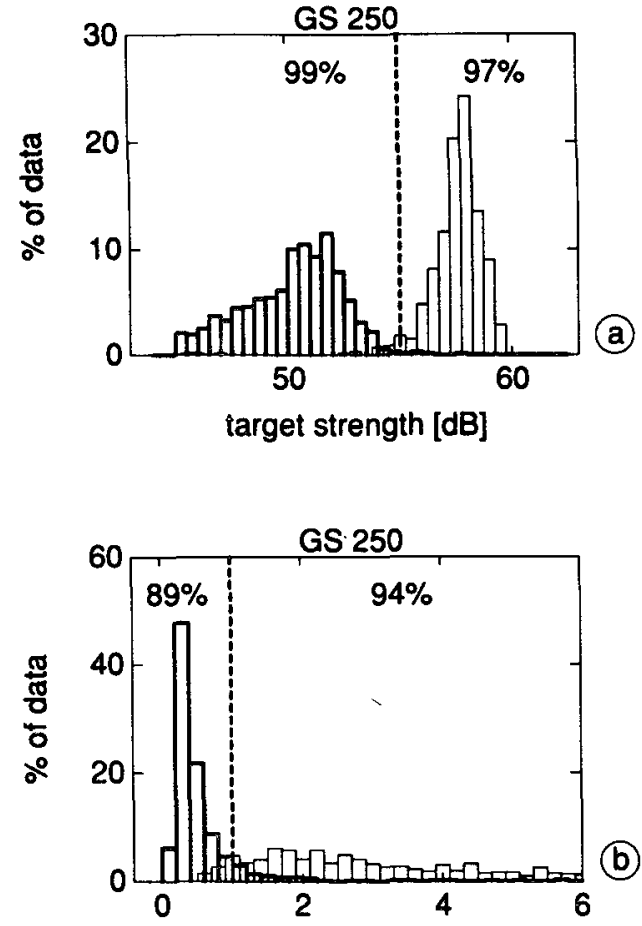

vertical velocity variance $\left[\mathrm{cm}^{\wedge} 2 / \mathrm{s}^{\wedge} 2\right]$
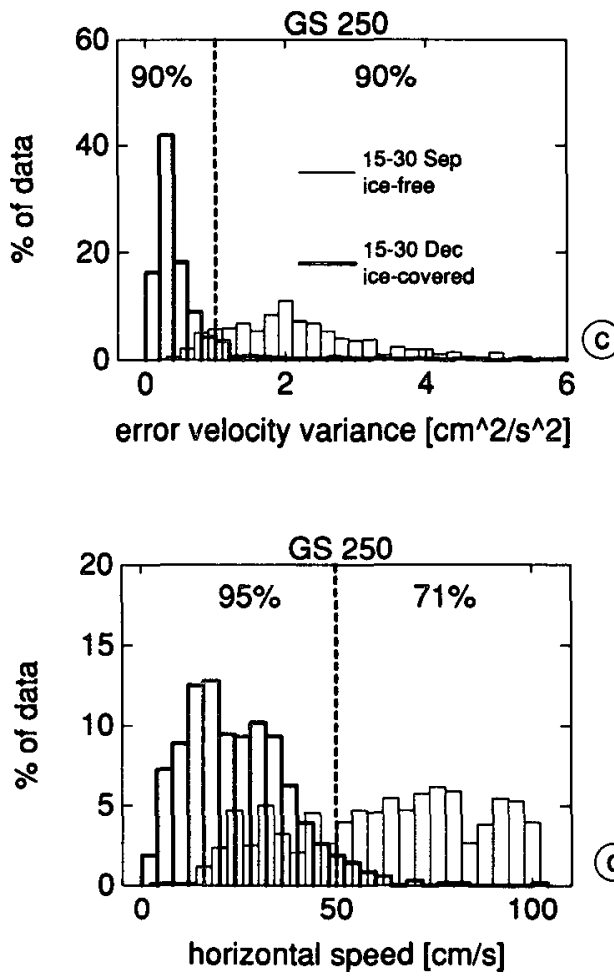

(d)

FIG. 9. Histogram distributions during ice-free (15-30 September 1989) and ice-covered (15-30 December) times at station 250 of (a) surface target strength; (b) vertical velocity variance; (c) error velocity variance; (d) horizontal Doppler speed. Threshold values and percentage of discrimination are included. track is needed, however, as the following comparison will show.

The surface Doppler speed was significantly correlated with the ECMWF wind speed (0.81 at station 319 , and somewhat less for 250 ), and the data resemble

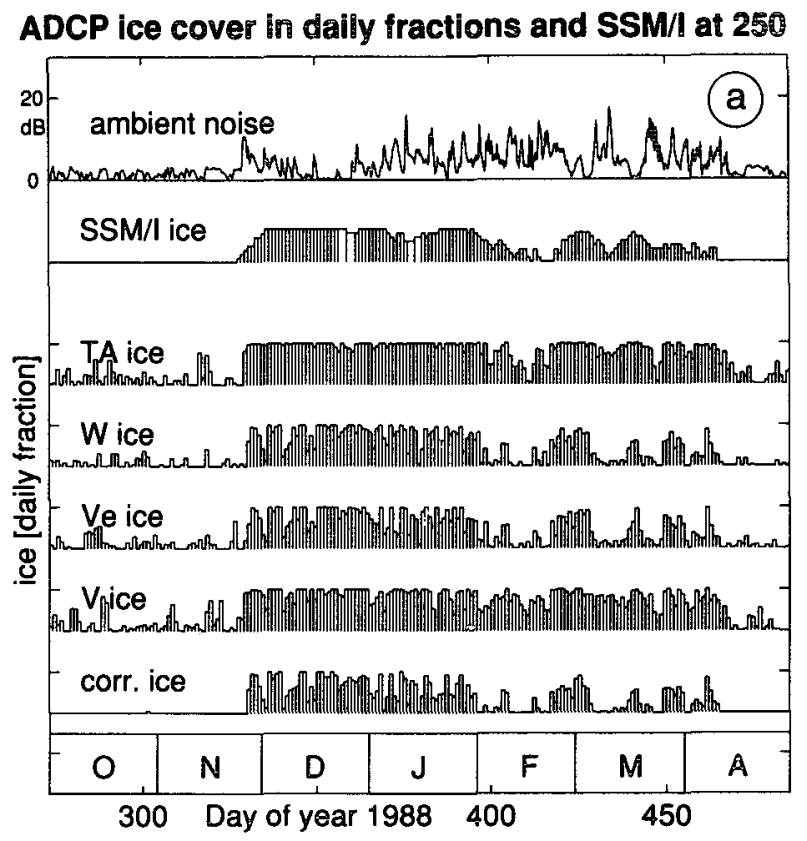

ADCP ice cover in daily fractions and SSM// at 319

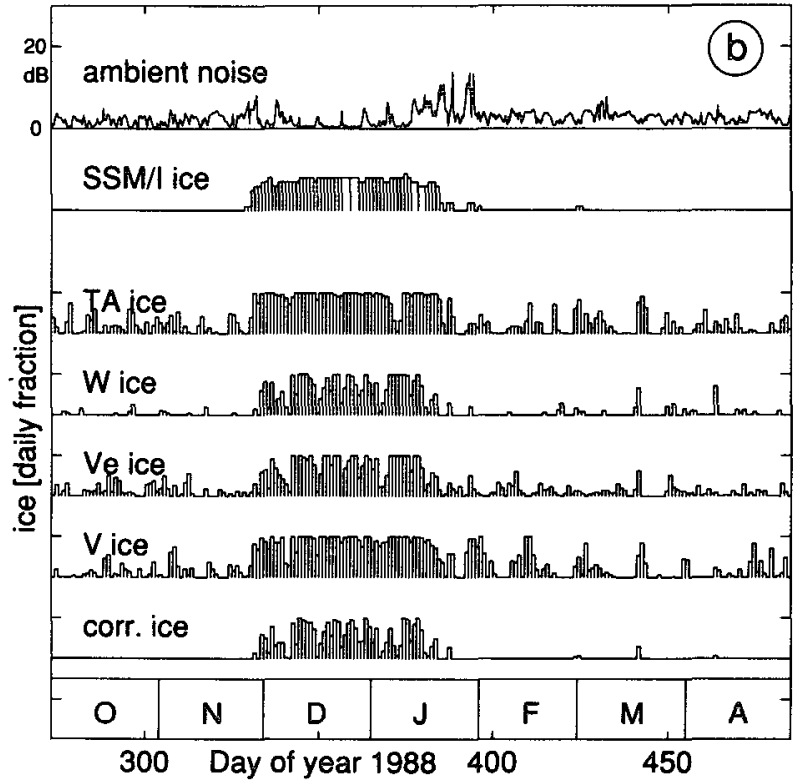

FIG. 10. Time series of ambient noise, SSM/I ice concentration and ADCP ice for (a) station 250 and (b) station 319. The ADCP ice is represented by individual parameters such as target strength TA vertical velocity variance $W$ error velocity variance $\mathrm{Ve}$, and horizontal speed $V$ and the correlation of the four (corr). 
the rule of the ice drift velocity being $1 / 50$ of the wind speed ( $1 / 52$ at mooring 250 and $1 / 59$ for 319 ).

The deflection angles between model winds and ADCP ice drift were small, which in part might be the consequence of the degraded quality of the direction measurements. At this high latitude the horizontal magnetic field intensity is relatively weak, and the internal flux gate compass of the ADCP showed significant deviations compared to the magnetic compass of the Aanderaa current meter moored below. We corrected for most of the differences using a heading-dependent deviation, but errors of some $10^{\circ}$ might still be present.

In general our finding is that the surface Doppler represents ice drift equally well to the bottom track used by Belliveau et al. (1990).

\section{3) ICE THICKNESS}

Adding a precise pressure sensor to the ADCP (which is available) should allow determination of ice drafts, similar to directly upward-looking sonars (Vinje and Berge 1989). The only pressure sensor we had was from an Aanderaa below the ADCP with a resolution of only $0.7 \mathrm{~m}$, which was not sufficient to measure ice drafts in this region.

A verification experiment using an ADCP with a high-precision pressure sensor and some independent information about ice thickness would be valuable.

\section{4) ICE NOISE}

Compared to wind-induced ambient noise (see previous section ), a much louder signal was recorded at times when the MIZ was in the vicinity of the moorings (Fig. 10). Different processes can contribute to ice noise: thermal ice cracking at decreasing temperatures, breakup of continuous ice, and movements of noncontinuous ice covers (Urick 1975). In our case the latter is most plausible. During times with maximum ice concentration, from late December until early January, the ambient noise level was at minimum, indicating that wind noise is reduced under a continuous ice cover. Within the open ice field of the "Is Odden," however, the ambient noise was at maximum (Fig. $10 \mathrm{~b}$ ), indicating collisions of ice floes during storms or swell. During the same experiment, ambient noise levels were recorded from tomographic receivers (Lynch et al. 1993). Collisions of ice floes were found to be responsible for the enhanced low-frequency noise in the $200-300-\mathrm{Hz}$ band as well. Since the sound decays rapidly for the high (ADCP) frequencies, we obtain a locally generated signal, while the low-frequency part of the noise spectrum can travel significant horizontal distances, that is, yielding a more integral result.

In summary, $\mathrm{ADCP}$ ambient noise records provide an additional source of information on sea ice conditions.

\section{Summary}

Three new aspects of surface conditions measured by upward-looking ADCPs were presented.

First, a valuable extension to the parameters measured by the ADCPs is the determination of the instrument depth at an estimated precision of $0.2 \mathrm{~m}$ at no additional cost. Although not proven here, due to the lack of a precise-enough reference pressure, ice thickness measurements seem possible. This, together with measured ice drift, would allow an estimate of freshwater transport due to drifting ice.

Second, ice-covered phases can be discriminated from open water by combining changes in the surface target strength, surface velocity speed, and vertical and error velocity variances without using the bottom-track mode. The daily fraction of ice-covered phases was in general agreement with SSM/I satellite ice concentrations. Higher temporal resolution can be obtained, however, from the local ADCP ice detection.

Finally, independent information about wind speed and ice conditions was gained from the ambient noise records. We were able to improve wind stress estimates using ambient noise in favor of the surface backscatter strength. Further, there is the potential to evaluate ambient noise with respect to ice-floe collisions within the MIZ.

Acknowledgments. We would like to thank J. Meincke and his mooring group for their help with the ADCP moorings, L. Toudal for kindly providing SSM/I ice concentrations, and $K$. Arpe who made the ECMWF data available. D. Farmer, F. Schott, and U. Send contributed with helpful discussions. This study was supported by the Bundesministerium für Forschung und Technologie (BMFT), Contracts MFG 0071-8, 03R 617, and 03PL002A.

\section{REFERENCES}

Barry, R. G., J. Maslanik, K. Steffen, R. L. Weaver, V. Troisi, D. J. Cavalieri, and S. Martin, 1993: Advances in sea-ice research based on remotely sensed passive microwave data. Oceanography, 6(1), 4-12.

Belliveau, D. J., G. L. Bugden, B. M. Eid, and C. J. Calnan, 1990: Sea ice velocity measurements by upward-looking Doppler current profilers. J. Atmos. Oceanic Technol., 7, 596-602.

Brown, J., E. D. Barton, A. Trasvina, H. S. Velez, P. M. Kosro, and R. L. Smith, 1992: Estimation of surface winds from upward looking acoustic Doppler current profilers. J. Geophys. Res., 97, $17925-17930$.

Hunkins, K., 1975: The oceanic boundary layer and stress beneath a drifting ice floe. J. Geophys. Res., 80, 3425-3433.

Joyce, T. M., D. S. Bitterman, Jr., and K. E. Prada, 1982: Shipboard acoustic profiling of upper ocean currents. Deep-Sea Res., 29, 903-913.

Lynch, J. F., H. X. Wu, R. Pawlowicz, P. Worcester, R. Keenan, H. Graber, O. Johannessen, P. Wadhams, and R. Shuchman, 1993: Ambient noise measurements in the $200-300-\mathrm{Hz}$ band from the Greenland Sea tomography experiment. J. Acoust. Soc. Amer, 94(2), 1015-1033.

RDI-Primer, 1989: Acoustic Doppler Current Profilers Principles of Operation: A Practical Primer. RD Instruments, $36 \mathrm{pp}$. 
Schott, F., 1986: Medium-range vertical acoustic Doppler current profiling from submerged buoys. Deep-Sea Res., 33(10), 12791292.

- 1989: Measuring winds from underneath the ocean surface by upward-looking acoustic Doppler current profilers. J. Geophys. Res., 94, 8313-8321.

,- M. Visbeck, and J. Fischer, 1993: Observations of vertical currents and convection in the central Greenland Sea during the winter of 1988/89. J. Geophys. Res., 98, 14 401-14 421.

Urick, R. J., 1983: Principles of Underwater Sound, 3d ed. McGrawHill, $423 \mathrm{pp}$.
Vagle, S., and D. M., Farmer, 1992: The measurement of bubblesize distributions by acoustical backscatter. J. Atmos. Oceanic Technol., 9, 630-644.

- W. G. Large, and D. M. Farmer, 1990: An evaluation of the WOTAN technique of inferring oceanic winds from underwater ambient sound. J. Atmos. Oceanic Technol., 7, 576-595.

Vinje, T., and T. Berge, 1989: Upward looking sonar recordings at $75^{\circ} \mathrm{N}-12^{\circ} \mathrm{W}$ from $22 \mathrm{June} 1987$ to $20 \mathrm{June} 1988$. Tech. Rep. 51. Norsk Polarinstitutt, Bergen, $52 \mathrm{pp}$.

Waddell, S. R., and D. M. Farmer, 1988: Ice breakup: Observations of the acoustic signal. J. Geophys. Res., 93(c3), 2333-2342. 